\title{
A socio-ecological analysis of risk, protective and promotive factors for the mental health of Burundian refugee children living in refugee camps
}

\author{
Florian Scharpf $^{1}{ }^{1} \cdot$ Getrude Mkinga $^{3} \cdot$ Faustine Bwire Masath $^{1,2} \cdot$ Tobias Hecker $^{1,4}$
}

Received: 18 June 2020 / Accepted: 13 September 2020 / Published online: 21 September 2020

(c) The Author(s) 2020

\begin{abstract}
Children and adolescents' mental health risk and resilience arise from a complex interplay of factors on several socioecological levels. However, little is known about the factors that shape the mental health of refugee youth living in refugee camps close to ongoing conflict. We conducted a cross-sectional study with a representative sample of 217 Burundian refugee children aged 7-15 and their mothers residing in refugee camps in Tanzania to investigate associations between risk, protective and promotive factors from various ecological levels (individual, microsystem, exosystem), and children's post-traumatic stress disorder (PTSD) symptoms, internalizing and externalizing problems, and prosocial behavior. Data were collected using structured clinical interviews and analyzed using multiple regression models. Exposure to violence across all contexts and engagement coping were risk factors for PTSD symptoms and internalizing problems, while only violence by mothers seemed to increase children's vulnerability for externalizing problems. A differential impact of violence exposures on prosocial behavior was observed. Higher-quality friendships appeared to protect youth from PTSD symptoms and externalizing problems, while they also promoted children's prosocial behavior, just as mothers' social support networks. Prevention and intervention approaches should integrate risk, protective and promotive factors for refugee youth's mental health across multiple ecological contexts and take into account context-specific and adaptive responses to war and displacement.
\end{abstract}

Keywords Refugee children $\cdot$ Ecological $\cdot$ Risk factors $\cdot$ Mental health $\cdot$ Resilience $\cdot$ Post-traumatic stress

\section{Introduction}

Refugee children and adolescents are at an increased risk of developing mental health problems due to their exposure to violence before their flight, potentially traumatizing experiences during their journey and daily stressors after their arrival in the host country $[1,2]$. Accordingly, high prevalence rates of trauma-related psychopathology, such

Florian Scharpf

florian.scharpf@uni-bielefeld.de

1 Department of Psychology, Bielefeld University, P. O. Box 100131, 33501 Bielefeld, Germany

2 Vivo International, Konstanz, Germany

3 Department of Educational Psychology and Curriculum Studies, Dar es Salaam University College of Education, Dar es Salaam, Tanzania

4 Department of Psychology, University of Zurich, Zurich, Switzerland as post-traumatic stress disorder (PTSD), internalizing problems (e.g., depression and anxiety), and externalizing problems (e.g., aggressive and antisocial behavior), have been found among refugee youth [3,4]. A better understanding of the factors which increase and alleviate refugee children's vulnerability for mental health problems is urgently needed to develop targeted prevention and intervention strategies [2].

Healthy child development has been widely depicted using ecological systems theory [5], which describes development as interactions between children and their social environment across five nested contexts: ontogenetic level (individual attributes), microsystem (family and peers), mesosystem (interactions between microsystems), exosystem (wider community context) and macrosystem (society and culture). Such a socioecological framework has been recently applied to conceptualize the factors that contribute to the mental health and well-being of refugee and other conflict-affected children $[2,6,7]$. On the one hand, the model's broad view takes into account the shattering direct 
and indirect impact of war and displacement on all aspects of children's worlds. On the other hand, the model enriches this focus on risk factors with a resilience perspective, which considers those ecological factors that may be related to reduced mental health problems, i.e. protective factors, and to increased adjustment, i.e. promotive factors [8]. From a socio-ecological perspective, these factors are expected to have both direct and indirect effects on children's mental health through their interrelations with factors from the same and other ecological levels [6].

On the ontogenetic level, a higher exposure to war-related traumatic events is a powerful risk factor for refugee children's mental health across settings [2,9]. Cumulative premigration trauma predicted higher levels of PTSD symptoms, depression, and anxiety in longitudinal studies with refugee minors in Norway [10] and Belgium [11]. Although the way refugee children cope with their adverse experiences is likely crucial for their mental health, there has been little research on the role of coping strategies [7]. In the coping literature, engagement coping strategies, including problemfocused coping, support seeking, emotion regulation and cognitive restructuring, have been generally associated with positive mental health outcomes [12]. In a study with waraffected Bosnian adolescents [13], a greater use of engagement strategies was associated with lower levels of PTSD symptoms. Syrian children who coped by acquiring social support and trying to reframe events also reported fewer PTSD symptoms [14]. However, greater use of problemfocused coping strategies was related to PTSD in Bosnian adolescents who were not entitled to asylum [15]. Consequently, evidence on the nature of engagement coping in terms of risk or resilience for refugee youth's mental health is still inconclusive and limited to PTSD as an outcome.

Within the microsystem, refugee parents' well-being and their parenting behaviors are likely to be important determinants of their children' adjustment $[9,16]$. Research in postconflict settings has established a link between war trauma, family violence against children, and children's mental health problems [17]. Recent studies found that refugee parents' mental health problems stemming from exposure to war trauma and post-migration stressors were associated with greater levels of harsh parenting, which were in turn related to children's emotional and behavioral problems $[18,19]$. Adolescents living in refugee camps in Uganda and Rwanda who reported higher exposure to physical, verbal, and sexual abuse also reported higher levels of anxiety and depression symptoms [20]. High-quality friendships, in contrast, have been shown to be an important protective factor for the mental health of children and adolescents facing adversity [21, 22]. Moreover, higher-quality relationships with peers have been linked to higher levels of adolescents' empathy [23] and prosocial behavior [24, 25]. For refugee children, higher self-reported support from friends was related to lower levels of mental health problems and better adjustment in a systematic review [9]. Higher levels of support by peers also decreased the association between stressful life events and anxiety symptoms in a more recent study with unaccompanied minors in Germany [26]. However, the association of refugee children's friendship quality with their mental health has not been investigated.

Focusing on the exosystem, research with non-refugee populations suggests a strong link between increased levels of violence within the wider community and youth's mental health problems, particularly PTSD symptoms and externalizing problems [27]. Higher exposure to community violence in the United States predicted higher levels of PTSD symptoms and risk behaviors as well as worse academic outcomes in Khmer refugee adolescents [28]. However, no study has investigated the association between refugee children's current exposure to community violence and their mental health. Given the dynamic interaction between the different socio-ecological contexts, refugee parents may draw on social resources within the community that also indirectly benefit the individual child. For instance, wider and higher-quality parental social support networks have been linked to better mental health outcomes in children and adolescents [29]. Refugee adolescents exhibited lower levels of psychopathology when they reported having families with wide kin contacts and mothers who often received visitors at home [30]. The association between parents' social networks and child mental health is likely to be mediated by the positive impact of emotional and instrumental support on parental well-being and family functioning [31]. There is also evidence suggesting that mothers' social network quality predicts adolescents' own prosocial behavior through mechanisms of social learning [32].

Although $80 \%$ of refugees flee to neighboring low- and middle-income countries where they usually settle in refugee camps (UNHCR, 2019), current evidence on risk and protective factors for refugee youth's mental health is largely based on studies conducted in high-income countries, such as Europe, North America, and Australia [2, 9]. This means that the lived realities of the majority of refugee children are not adequately represented by the current state of research. While refugee children in high-income countries may have to cope primarily with adjustment to a different host culture and complex asylum processes, those resettling in camps in low-income settings often suffer from material hardships and face ongoing threats to their security [2]. Among the studies that investigated risk and protective factors associated with the mental health of refugee youth in camps, most focused on individual-level factors and few considered the family and community context [4]. Another shortcoming of available studies is the unilateral focus on negative mental health outcomes. To understand children's resilience in the face of severe adversity, it is also important to look at positive 
aspects of mental health and to identify factors which promote these outcomes [8].

Applying a socio-ecological framework, this study aimed to investigate risk, protective, and promotive factors for the mental health of Burundian refugee children and adolescents currently living in refugee camps. In 2015, opposition against the Burundian president's illegitimate third term led members of the ruling party to commit violence and atrocities towards perceived opponents, including abductions, extrajudicial killings, and torture, and caused more than three hundred thousand Burundians to flee to neighbouring countries [33]. Based on available evidence, we expected that exposure to violence on the different ecological levels (war exposure, violence by mothers, community violence) would be associated with higher levels of mental health problems and lower levels of prosocial behavior. Due to the inconclusive findings, we did not have a priori hypotheses regarding the association between engagement coping and mental health outcomes. Based on previous findings with refugee and non-refugee youth, we expected that higherquality relationships with friends as well as higher quality maternal social support networks would be associated with lower levels of mental health problems and higher levels of prosocial behavior.

\section{Methods}

\section{Sampling and participants}

The study was conducted between January and May 2018 in three large refugee camps (Nduta, Nyarugusu, and Mtendeli) in Western Tanzania. Recruitment was conducted using a combined systematic and random sampling approach to identify family triads consisting of the mother, the father, and the oldest child between 7 and 15 years, i.e. of primary school age (for details see [34]). For the current study, we focused on children and their mothers because women are primarily in charge of children's socialization in Burundian culture [35]. The final study sample consisted of 217 children $(47 \%$ female) and their mothers. As $85.6 \%(n=186)$ of the primary female caregivers were children's biological mothers, we refer to them as mothers. Other types of caregiver were relatives, such as aunts or sisters $(5.6 \%, n=12)$, step mothers $(2.8 \%, n=6)$, and foster mothers $(6 \%, n=13)$. The majority of the families $(65.9 \%, n=143)$ arrived at the camps in 2015 after the outbreak of political violence in Burundi. Most mothers $(79.7 \%, n=173)$ identified the political conflicts as the main reason for their flight. Table 1 shows other sociodemographic characteristics of the children and mothers.

\section{Procedure}

Data collection took place on the compounds of collaborating NGOs within the camps. Upon arrival, families were provided information regarding the purpose and the procedure of the study. Then, each family member gave their informed consent by signing with their names or fingerprints. Parents gave their consent on behalf of children under the age of 11 . All but two families were willing to participate in the study. The research team, consisting of Tanzanian master-level psychologists and trained research assistants from the refugee community residing in the camps, conducted structured
Table 1 Sociodemographic characteristics of children and mothers

\begin{tabular}{lcc}
\hline & Children $(n=217)$ & Mothers $(n=217)$ \\
\hline Age in years, $M$ (SD, range) & $12.16(2.03,7-15)$ & $34.74(8.52,19-74)$ \\
Education, \% $(n)$ & & \\
No schooling & $8.3(18)$ & $35.0(76)$ \\
Class 1-3 (primary) & $49.3(97)$ & $30.8(67)$ \\
Class 4-6 & $39.6(86)$ & $10.7(23)$ \\
Some secondary & $2.8(6)$ & \\
Completed secondary ${ }^{\text {a }}$ & & \\
Ethnicity & & \\
Hutu & $82.5(179)$ \\
Tutsi & $14.4(31)$ & \\
Other & $3.1(7)$ & \\
Household size, $M$ (SD, range) & $7.3(1.98,3-14)$ & \\
Household income $p$. month (USD), $\%(n)$ & & \\
No income & $36.4(79)$ & \\
Up to 20 & $54.9(119)$ & \\
More than 20 & $8.7(19)$ & \\
\hline
\end{tabular}

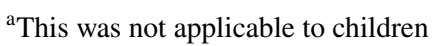


clinical interviews individually with each parent and child in Swahili, the lingua franca in Tanzania and the refugee camps, or in Kirundi, the native language of Burundians. Trained interpreters were always present in case a participant was not proficient in Swahili (for details on the procedure see [34]). After the interviews, families received a material compensation of 8 US Dollars. The study was approved by the Ethics Commission of the University of Zurich and the Tanzanian National Institute for Medical Research. All necessary research permits were obtained from the Tanzanian Commission for Science and Technology (COSTECH) and the Tanzanian Ministry of Home Affairs.

\section{Measures}

The interview guides for mothers and children consisted of individual questionnaires that were either already available in Swahili or were translated from English to Swahili for this study using blind-back translation [36]. Prior to data collection, we conducted focus group discussions with the Burundian research assistants to qualitatively evaluate the appropriateness of the instruments and relevant mental health concepts in Burundian culture. We also conducted a pilot assessment with eight families, which allowed us to make necessary adaptations and supported the appropriateness of the instruments for the study context.

\section{Sociodemographic information}

Children and mothers were asked about their age and educational level. Mothers also answered questions about their ethnicity, date of arrival at the camp, household characteristics (size, average income per month) and the reasons for their flight.

\section{Post-traumatic stress symptoms}

The University of California at Los Angeles Child/Adolescent PTSD Reaction Index for DSM-5 (PTSD-RI-5) [37] was used to assess children's PTSD symptoms within the past month according to the criteria of the Diagnostic and Statistical Manual of Mental Disorders (DSM) 5th Edition [38]. The 31 items are scored on a 5-point Likert scale ranging from 0 (none of the time) to 4 (most of the time) and are summed up to a total score of PTSD symptom severity ranging from 0 to 124 . The PTSD-RI-5 has been used in various cultural settings and has shown good psychometric properties [39]. It has also been applied successfully in studies with refugee youth [40]. Internal consistency of the total score was high in our sample, with Cronbach's $\alpha=0.90$.
Internalizing and externalizing problems and prosocial behaviour

The self-report version of the Strength and Difficulties Questionnaire (SDQ) is a widely used measure to screen for emotional and behavioural problems in children and adolescents with good psychometric properties [41]. The 25 items are rated as not true (0), somewhat true (1), or certainly true (2). All items are divided between five subscales of five items each: emotional problems, peer problems, hyperactivity/inattention, conduct problems, and prosocial behavior. The subscales emotional problems and peer problems can be combined to create an internalizing problem score (range 0-20), while the sum of the subscales hyperactivity/inattention and conduct problems yields an externalizing problems score (range $0-20$ ). The four subscales combined provide an index of total difficulties (range 0-40). The SDQ has been extensively used in Sub-Saharan Africa [42] and in refugee settings [16, 20]. Cronbach's alpha of the total difficulties score was 0.65 in our sample, which is comparable to other studies [16, 43]. The rather low internal consistency can be attributed to the heterogeneity of the total score consisting of both internalizing and externalizing symptoms across four subscales.

\section{War-related traumatic events}

22 items derived from a checklist by Neuner et al. [44] were used to assess children's exposure to war-related traumatic events (e. g. physical injury, sexual assault, dangerous flight).

\section{Engagement coping}

We used an adapted version of the Kidcope [45] that had been developed based on qualitative research with waraffected Congolese adolescents [46] to assess the frequency of children's use of engagement coping strategies. It covered five engagement strategies (cognitive restructuring, social support, problem solving, emotion regulation, praying) and six disengagement strategies (withdrawal, distraction, wishful thinking, self-criticism, resignation, blaming others) with 18 items. Similar to previous work [46], we asked children first to identify one stressful situation within the past week, for example in their family, with friends, or at school, and then to indicate whether they had used a specific strategy to cope with that situation on a binary yes (1)/no (0) scale. Here, we were only interested in the five engagement strategies measured by seven items (range 0-7). 


\section{Violence by mothers}

We used the Parent-Child Conflict Tactic Scales (CTSPC) [47] to assess the mother's use of physical and emotional violence towards the participating child. Children reported the frequency of specific abusive acts within the past year over 18 items on a 7-point Likert scale ranging from $0=$ never to $25=>20$ times. Summing up all items yielded a total score of maternal violence within the past year with a potential range from 0 to 450 . The CTSPC self-report version has shown good psychometric properties [48] and has been used successfully in East Africa [49]. Internal consistency of the total violence score was $\operatorname{good}(\alpha=0.80)$.

\section{Quality of relationships with friends}

A shortened version [50] of the People in My Life Questionnaire (PIML) [51] was used to assess the quality of children's relationships with their friends. The PIML was originally designed to measure children's internal representations of their relationship with parents, friends and teachers. The 15 items referring to friends are rated on a 4-point scale from 0 (almost never or never true) to 3 (almost always or always true) and cover the subscales trust, communication and alienation [52]. The PIML has demonstrated good psychometric properties [52, 53]. Cronbach's Alpha of the total score ranging from 0 to 45 was good $(\alpha=0.82)$.

\section{Community violence}

Mothers' report of experienced and witnessed violence committed by community members within the past month served as a proxy for children's current exposure to community violence. The nine items answered on a binary yes (1)/no (0) scale covered different violence-related events, e.g., physical and sexual assault, and were derived from a checklist used in a study with Congolese refugees in a Ugandan refugee camp [54].

\section{Maternal social support networks}

Five purpose-built items assessed how often mothers had met with others in a public place, received visitors at home, visited other people and talked about problems with friends, family members or other persons with similar traumas within the past month. The items were rated on a 5-point scale from never (0) to very often (4) and summed up to receive a total score ranging from 0 to 20 . Internal consistency was acceptable $(\alpha=0.71)$.
Table 2 Descriptive statistics of study variables

\begin{tabular}{ll}
\hline Study variables, $M$ (SD, min-max) & \\
Posttraumatic stress symptoms (UCLA-RI-5) & $14.98(11.31,0-49)$ \\
Internalizing problems (SDQ) & $6.36(3.24,0-16)$ \\
Externalizing problem (SDQ) & $4.40(2.93,0-15)$ \\
Prosocial behavior (SDQ) & $8.26(1.57,3-10)$ \\
War-related traumatic events & $3.77(3.68,0-16)$ \\
Engagement coping (Kidcope) & $3.87(1.90,0-7)$ \\
Violence by mothers (CTSPC) & $42.91(42.28,0-214)$ \\
Friendship quality (PIML) & $31.43(8.02,5-45)$ \\
Community violence & $2.67(2.46,0-9)$ \\
Maternal social support network & $9.47(5.00,0-20)$ \\
\hline
\end{tabular}

UCLA-RI-5 University of California at Los Angeles Child/Adolescent PTSD Reaction Index for DSM-5, SDQ Strengths and Difficulties Questionnaire, CPCTS Parent-Child Conflict Tactic Scales, PIML People in My Life Questionnaire

\section{Data analysis}

The data were analysed using the Statistical Package for the Social Sciences (SPSS) version 25.0. There were no missing values. To investigate the associations between presumed risk, protective and promotive socio-ecological factors, and the outcomes PTSD, internalizing problems, externalizing problems, and prosocial behaviour, we conducted four multiple linear regression models, one for each outcome. The predictors on the different ecological levels - exposure to war-related trauma and engagement coping on the ontogenetic level, violence by mothers and friendship quality in the microsystem, community violence and mothers' social network in the exosystem - were entered at once in the regression models.

We retained univariate outliers in the analyses to also consider naturally occurring extreme values in children's experiences and mental health. Multivariate outliers were removed from regression analyses. The regression models fulfilled all other necessary quality criteria for linear regression analysis. Residuals did not deviate significantly from normality, linearity, or homoscedasticity. The maximum variance inflation factor for the regression models did not exceed 1.26. Hence, we did not need to take multicollinearity into account. Children's age and gender were added as covariates in the regression models due to their potential impact on both outcome variables and predictor variables [16]. Our metric for a small effect size was $f^{2} \geq 0.02$, for a medium effect $f^{2} \geq 0.15$, and for a large effect $f^{2} \geq 0.35$ [55]. All analyses used a two-tailed $\alpha=0.05$.

\section{Results}

Descriptive statistics and bivariate correlations are displayed in Tables 2 and 3. Table 4 shows the results of the multiple regression analyses. The model predicting PTSD symptoms 
explained $41 \%$ of the variability of PTSD symptoms [adj. $\left.R^{2}=0.41, F(8,198)=18.83, p<0.001, f^{2}=0.69\right]$. Exposure to war-related traumatic events, violence by mothers and community violence as well as engagement coping strategies were significantly positively associated with PTSD symptoms, whereas friendship quality was significantly negatively related to PTSD symptoms. The model predicting internalizing problems explained $12 \%$ of the variability of internalizing problems [adj. $R^{2}=0.12, F(8,194)=4.46, p<0.001$, $\left.f^{2}=0.14\right]$. Exposure to war-related traumatic events, violence by mothers and community violence as well as engagement coping strategies were significantly positively associated with internalizing problems. The model predicting externalizing problems explained $12 \%$ of the variability of externalizing problems [adj. $R^{2}=0.12, F(8,191)=4.29, p<0.001$, $\left.f^{2}=0.14\right]$. Exposure to violence by mothers was significantly positively associated and friendship quality was significantly negatively associated with externalizing problems. Finally, the model predicting prosocial behavior accounted for $19 \%$ of the variability in prosocial behavior [adj. $R^{2}=0.19, F(8$, $\left.200)=7.20, p<0.001, f^{2}=0.23\right]$. Exposure to war-related and community violence, friendship quality and the quality of mothers' social networks were significantly positively related to prosocial behavior, whereas violence by mothers was significantly negatively related to prosocial behavior.

\section{Discussion}

This study investigated risk, protective, and promotive factors across multiple socio-ecological levels (individual level, microsystem, exosystem) for negative (PTSD symptoms, internalizing and externalizing problems) and positive (prosocial behavior) mental health outcomes in Burundian refugee youth living in camps close to ongoing conflict. As expected, higher exposure to violence related to war, within the family, and in the community was associated with higher levels of PTSD symptoms and internalizing problems. This is consistent with a large body of evidence documenting the detrimental mental health impact of traumatic experiences $[2,56]$, child maltreatment $[20,40,57]$ and community violence $[27,28]$ for refugee and non-refugee children and adolescents across various socioeconomic and cultural settings. Studies conducted within a socio-ecological framework have shown how violence on more distal ecological levels, e.g., structural and community violence, adversely affects children's adjustment both directly and indirectly by increasing violence within the more proximal family context [58-60].

The unexpected findings that higher exposure to warrelated and community violence was not associated with externalizing problems and with higher levels of prosocial behavior are noteworthy. This contradicts empirical findings and theoretical conceptualizations of how organized

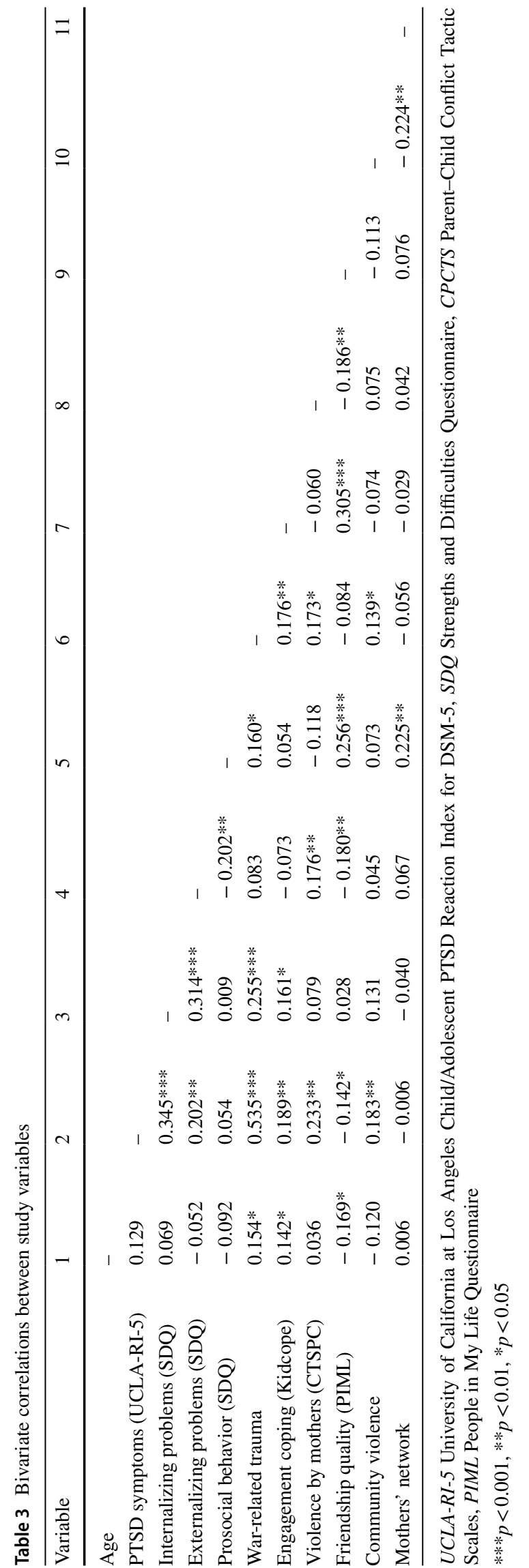




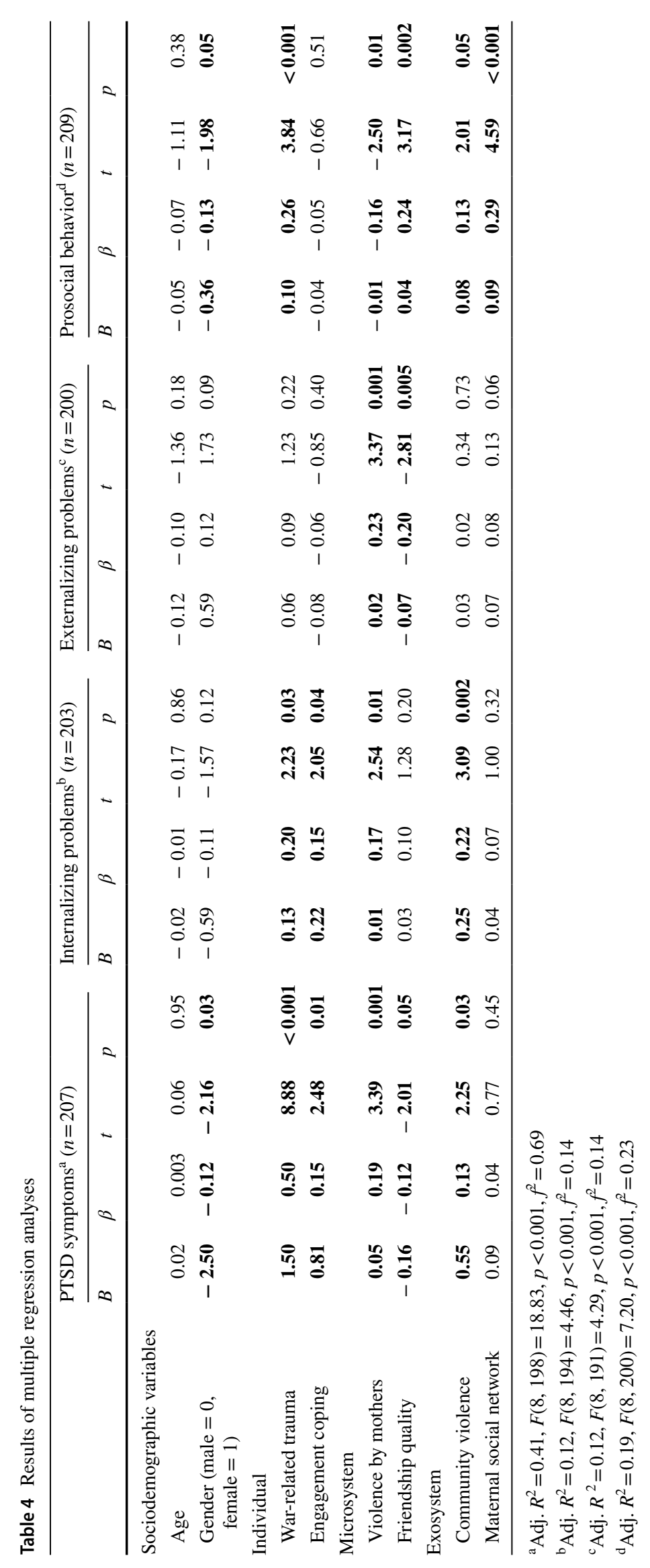


and community violence are associated with aggressive and antisocial tendencies and undermine prosocial behavior [54, 61-65]. However, preliminary evidence suggests that victimization and war trauma may also favor prosocial behaviors and orientations through positive experiences in the midst of adversity, e.g., receiving and giving care and help or learning from prosocial role models, thereby reflecting resilience [66]. A more prosocial attitude may be adaptive in times of violent intergroup conflict as cooperative group members may be more likely to be rewarded and non-cooperative members to be punished [67]. The finding fits with the observed lack of an association between both war-related and community violence with externalizing problems, which also include antisocial behaviors and may, thus, be considered the converse of prosocial behavior. In contrast, increased violence by mothers was associated with increased externalizing problems and reduced prosocial behavior, suggesting the importance of social learning through parental role models for both antisocial and prosocial behavior $[62,68]$. The pattern of associations between violence on different ecological contexts, externalizing, and prosocial behaviors is in line with a recent study with unaccompanied refugee minors, which found family violence, but not organized violence, to be associated with self-reported aggression [69].

A more frequent use of engagement coping strategies in everyday situations, such as problem-focused coping and cognitive restructuring, was associated with higher levels of PTSD symptoms and internalizing problems, which is inconsistent with studies that found engagement strategies to be related to lower levels of mental health problems in refugee children and adults [13, 14, 70-72]. However, other studies found that more problem-focused coping was associated with higher levels of internalizing problems in sample of adult Syrian refugees living in Turkey close to the Syrian border [73] and with the presence of PTSD in Bosnian refugee youth waiting for the resolution of their asylum claims [15]. Our finding adds to these and suggests that engaging intensively with situations and stressors that cannot be easily changed or controlled may also be detrimental to mental health. The children in our study lived in refugee camps close to ongoing conflict and under precarious circumstances. Many of the stressful situations they had to cope with referred to experiencing or witnessing violence at home, in the community, or at school, which may have been perceived as being beyond the children's ability to control.

In the face of severe adversity, children with closer and more supportive relationships with friends reported lower levels of PTSD symptoms and externalizing problems as well as higher levels of prosocial behavior. This is in keeping with studies with non-refugee youth showing that higherquality peer relationships moderated the impact of complex trauma [22], family adversity [74], and peer rejection [21] on youth's adjustment. The finding is also consistent with previous evidence on the protective role of peer support for refugee children and adolescents' mental health $[9,26]$. The positive association between friendship quality and prosocial behavior points to the importance of peer relationships for the development of prosocial behavior [68]. Higher-quality maternal social support networks appeared to have a similarly promotive impact on children's prosocial behavior. This may be explained through social learning mechanisms, such as social behaviors, modeled by mothers in their interactions that are observed and imitated by children [32]. However, we did not observe the expected associations between maternal social network and children's mental health problems that has been found in previous studies [29, 30]. It is possible that a wider and closer social network of mothers indirectly exposes youth to the traumas and problems of other community members, which may outweigh any positive indirect impact of maternal social networks for youth's mental health. However, this hypothesis requires further testing.

The current study's novel contributions to the evidence base on refugee children and adolescents' mental health are threefold. First, the present study contributes to efforts to better understand and support the mental health and well-being of refugee youth living in refugee camps close to ongoing conflict, a population that represents the majority of refugee minors globally. Second, the study shows that risk, protective, and promotive factors from different levels in children's social environment are relevant for their adjustment and, thus, strengthens the importance of a socio-ecological perspective in research and practical work with refugee children. Third, the study not only includes a range of different negative mental health outcomes, but also investigates prosocial behavior reflecting positive adjustment. However, some limitations must be noted. First, due to the cross-sectional study design causal interpretations of the observed associations should be made with caution. Reciprocity may also be plausible when interpreting the present findings. For instance, children's externalizing problems have been shown to elicit physical discipline by parents [75], while more prosocial behavior also led to increased acceptance by peers [76]. Second, the specific cultural background and living context of our sample may limit the generalizability of our findings to refugee youth with other backgrounds or living in other settings, e.g., in high-income countries or urban community settings. However, we argue that the factors we included in our study are also relevant and salient in the social ecologies of children living in refugee camps in other areas of the world and, thus, assume a certain generalizability to these contexts, which nevertheless has to be examined in future studies. Third, although we ensured the suitability of our measures through focus group discussions and a pilot assessment, they have not been validated 
for our study sample. We recognize the importance of validating assessment tools for refugee youth in diverse settings [77], and hope that future research can address this shortcoming. Fourth, we assessed children's prosocial behavior through their self-report, which may be affected by social desirability. Direct ratings by peers or parents may also be valuable to include for analysis. Relatedly, we used maternal reports as a proxy for children's exposure to community violence which may underestimate the amount of children's actual exposure. The assessment of community factors, such as violence or social support, from the child perspective or through more objective indicators may be useful in future studies.

Our findings may have important implications for the practical clinical work with refugee youth in camp settings. Interventions should target factors on different ecological levels, i.e. related to the individual child, family, peers and the community, and various mental health outcomes, i.e. negative and positive aspects of adjustment. Considering the strong detrimental impact of war-related trauma and ongoing violence, we advocate programs with a trauma focus. As violence by mothers broadly impairs children's mental health, approaches should include parents and provide access to non-violent parenting strategies. The potentially protective and promotive nature of positive peer relationships may be harnessed by also considering interpersonal relationship skills as targets for intervention, incorporating psychosocial recreational elements (e.g., child-friendly spaces within the refugee camp), and by the choice of setting, for example, conducting interventions in a group format or in a school setting. Utilizing existing community spaces and incorporating lay personnel into care provision would also require fewer additional resources in camp settings that face severe resource deficits. Examples for possible interventions include trauma-focused cognitive behavioral therapy [78] and its adaption teaching recovery techniques [79], which have been tested in studies with war-affected Congolese girls [80] and unaccompanied refugee minors in Sweden [81], respectively. These programs also focus on coping with trauma and distress. Most notably, our findings suggest that it is important to take into account the contextdependent nature of certain coping styles and to carefully evaluate whether these may be adaptive or maladaptive for youth. Similarly, potential positive outcomes in the face of trauma and violence, such as an increased prosocial orientation, need to be considered by practitioners working in prevention and intervention.

In conclusion, exposure to violence across all contexts and engagement with everyday stressors appears to increase refugee youth's risk for mental health problems, but there is also evidence for peer and community resources contributing to resilience in the midst of war and displacement.
Prevention and intervention approaches aiming to improve and promote refugee children's mental health should focus on reducing children's exposure to violence in their social ecology and strengthen their relationships with peers.

Acknowledgements This research was supported by the North-South Cooperation at Zurich University (F-63212-13-01). We would like to thank all participating families in the refugee camps. Special thanks go to Plan International Tanzania and International Rescue Committee Tanzania for providing space and resources for data collection. We are extremely grateful to our highly motivated research team, to Edna Kyaruzi (M. A.), Mabula Nkuba (Ph.D.) and Maregesi Machumu (Ph. D.), Dar es Salaam University College of Education, and to Markus Landolt (Ph.D.) and Andreas Maercker (Ph.D., MD), University of Zurich, for their continuous support.

Funding Open Access funding enabled and organized by Projekt DEAL.

\section{Compliance with ethical standards}

Conflict of interest On behalf of all authors, the corresponding author states that there is no conflict of interest.

Ethical standards statement The study has received ethical approval by the ethics committee of the University of Zurich, Switzerland, and of the National Institute for Medical Research, Tanzania, and has therefore been performed in accordance with the ethical standards laid down in the 1964 Declaration of Helsinki and its later amendments.

Open Access This article is licensed under a Creative Commons Attribution 4.0 International License, which permits use, sharing, adaptation, distribution and reproduction in any medium or format, as long as you give appropriate credit to the original author(s) and the source, provide a link to the Creative Commons licence, and indicate if changes were made. The images or other third party material in this article are included in the article's Creative Commons licence, unless indicated otherwise in a credit line to the material. If material is not included in the article's Creative Commons licence and your intended use is not permitted by statutory regulation or exceeds the permitted use, you will need to obtain permission directly from the copyright holder. To view a copy of this licence, visit http://creativecommons.org/licenses/by/4.0/.

\section{References}

1. Hebebrand J, Anagnostopoulos D, Eliez S, Linse H, Pejovic-Milovancevic M, Klasen H (2016) A first assessment of the needs of young refugees arriving in Europe: what mental health professionals need to know. Eur Child Adolesc Psychiatry 25(1):1-6. https ://doi.org/10.1007/s00787-015-0807-0

2. Reed RV, Fazel M, Jones L, Panter-Brick C, Stein A (2012) Mental health of displaced and refugee children resettled in lowincome and middle-income countries: risk and protective factors. Lancet 379(9812):250-265. https://doi.org/10.1016/S0140 $-6736(11) 60050-0$

3. Kien C, Sommer I, Faustmann A et al (2019) Prevalence of mental disorders in young refugees and asylum seekers in European countries: a systematic review. Eur Child Adolesc Psychiatry. https:// doi.org/10.1007/s00787-018-1215-Z 
4. Vossoughi N, Jackson Y, Gusler S, Stone K (2018) Mental health outcomes for youth living in refugee camps. Trauma Violence Abus 19(5):528-542. https://doi.org/10.1177/1524838016673602

5. Bronfenbrenner U (1979) The ecology of human development: experiments by nature and design. Harvard University Press, Cambridge

6. Betancourt TS, Khan KT (2008) The mental health of children affected by armed conflict: protective processes and pathways to resilience. Int Rev Psychiatry 20(3):317-328. https://doi. org/10.1080/09540260802090363

7. Eruyar S, Huemer J, Vostanis P (2018) Review: How should child mental health services respond to the refugee crisis? Child AdolescMent Health 23(4):303-312. https://doi.org/10.1111/ camh. 12252

8. Tol WA, Song S, Jordans MJD (2013) Annual research review: resilience and mental health in children and adolescents living in areas of armed conflict-a systematic review of findings in lowand middle-income countries. J Child Psychol Psychiatry Allied Discip 54(4):445-460. https://doi.org/10.1111/jcpp.12053

9. Fazel M, Reed RV, Panter-Brick C, Stein A (2012) Mental health of displaced and refugee children resettled in high-income countries: risk and protective factors. Lancet 379(9812):266-282. https ://doi.org/10.1016/S0140-6736(11)60051-2

10 Jensen TK, Skar A-MS, Andersson ES, Birkeland MS (2019) Long-term mental health in unaccompanied refugee minors: preand post-flight predictors. Eur Child Adolesc Psychiatry. https:// doi.org/10.1007/s00787-019-01340-6

11. Vervliet M, Lammertyn J, Broekaert E, Derluyn I (2014) Longitudinal follow-up of the mental health of unaccompanied refugee minors. Eur Child Adolesc Psychiatry 23:337-346. https://doi. org/10.1007/s00787-013-0463-1

12. Carver CS, Connor-Smith J (2010) Personality and coping. Annu Rev Psychol 61(1):679-704. https://doi.org/10.1146/annur ev.psych.093008.100352

13. Howell KH, Kaplow JB, Layne CM et al (2015) Predicting adolescent posttraumatic stress in the aftermath of war: Differential effects of coping strategies across trauma reminder, loss reminder, and family conflict domains. Anxiety Stress Coping 28(1):88-104. https://doi.org/10.1080/10615806.2014.910596

14. Khamis V (2019) Posttraumatic stress disorder and emotion dysregulation among Syrian refugee children and adolescents resettled in Lebanon and Jordan. Child AbusNegl 89:29-39. https:// doi.org/10.1016/j.chiabu.2018.12.013

15. Elklit A, OstergardKjaer K, Lasgaard M, Palic S (2012) Social support, coping and posttraumatic stress symptoms in young refugees. Torture 22(1):11-23

16 Eruyar S, Maltby J, Vostanis P (2018) Mental health problems of Syrian refugee children: the role of parental factors. Eur Child Adolesc Psychiatry. https://doi.org/10.1007/s00787-017-1101-0

17. Catani C (2018) Mental health of children living in war zones: a risk and protection perspective. World Psychiatry 17(1):104-105. https://doi.org/10.1002/wps.20496

18. Sim A, Bowes L, Gardner F (2018) Modeling the effects of war exposure and daily stressors on maternal mental health, parenting, and child psychosocial adjustment: a cross-sectional study with Syrian refugees in Lebanon. Glob Ment Heal 5:e40. https://doi. org/10.1017/gmh.2018.33

19. Bryant RA, Edwards B, Creamer M et al (2018) The effect of post-traumatic stress disorder on refugees' parenting and their children's mental health: a cohort study. Lancet Public Health 3(5):e249-e258. https://doi.org/10.1016/S2468-2667(18)30051-3

20 Meyer SR, Yu G, Hermosilla S, Stark L (2017) Latent class analysis of violence against adolescents and psychosocial outcomes in refugee settings in Uganda and Rwanda. Glob Ment Health 4:e19. https://doi.org/10.1017/gmh.2017.17
21. Waldrip AM, Malcolm KT, Jensen-Campbell LA (2008) With a little help from your friends: the importance of high-quality friendships on early adolescent adjustment. Soc Dev 17(4):832852. https://doi.org/10.1111/j.1467-9507.2008.00476.x

22. Yearwood K, Vliegen N, Chau C, Corveleyn J, Luyten P (2019) When do peers matter? The moderating role of peer support in the relationship between environmental adversity, complex trauma, and adolescent psychopathology in socially disadvantaged adolescents. J Adolesc 72:14-22. https://doi.org/10.1016/j.adolescenc e.2019.02.001

23. Boele S, Van der Graaff J, de Wied M, Van der Valk IE, Crocetti E, Branje S (2019) Linking parent-child and peer relationship quality to empathy in adolescence: a multilevel meta-analysis. J Youth Adolesc 48(6):1033-1055. https://doi.org/10.1007/s1096 4-019-00993-5

24. Laible D (2007) Attachment with parents and peers in late adolescence: links with emotional competence and social behavior. PersIndivid Differ 43(5):1185-1197. https://doi.org/10.1016/j. paid.2007.03.010

25. Padilla-Walker LM, Fraser AM, Black BB, Bean RA (2015) Associations between friendship, sympathy, and prosocial behavior toward friends. J Res Adolesc 25(1):28-35. https://doi. org/10.1111/jora.12108

26. Sierau S, Schneider E, Nesterko Y, Glaesmer H (2018) Alone, but protected? Effects of social support on mental health of unaccompanied refugee minors. Eur Child Adolesc Psychiatry. https ://doi.org/10.1007/s00787-018-1246-5

27. Fowler PJ, Tompsett CJ, Braciszewski JM, Jacques-Tiura AJ, Baltes BB (2009) Community violence: a meta-analysis on the effect of exposure and mental health outcomes of children and adolescents. Dev Psychopathol 21(1):227-259. https://doi.org/10.1017/ S0954579409000145

28. Berthold SM (2000) War traumas and community violence: psychological, behavioral, and academic outcomes among khmer refugee adolescents. J MulticultSoc Work 8(1-2):15-46. https:// doi.org/10.1300/J285v08n01_02

29. McPherson KE, Kerr S, McGee E et al (2014) The association between social capital and mental health and behavioural problems in children and adolescents: an integrative systematic review. BMC Psychol 2(1):7. https://doi.org/10.1186/2050-7283-2-7

30. Tousignant M, Habimana E, Biron C, Malo C, Sidoli-LeBlanc E, Bendris N (1999) The Quebec adolescent refugee project: psychopathology and family variables in a sample from 35 nations. J Am Acad Child Adolesc Psychiatry 38(11):1426-1432. https:// doi.org/10.1097/00004583-199911000-00018

31. Alvarez EC, Kawachi I, Romani JR (2017) Family social capital and health-a systematic review and redirection. Sociol Health Illn 39(1):5-29. https://doi.org/10.1111/1467-9566.12506

32. Markiewicz D, Doyle AB, Brendgen M (2001) The quality of adolescents' friendships: associations with mothers' interpersonal relationships, attachments to parents and friends, and prosocial behaviors. J Adolesc 24(4):429-445. https://doi.org/10.1006/ jado.2001.0374

33. Human Rights Watch (2017) World Report 2017: Burundi. https ://www.hrw.org/world-report/2017/country-chapters/burundi. Accessed 14 Nov 2018

34. Scharpf F, Kyaruzi E, Landolt MA, Hecker T (2019) Prevalence and co-existence of morbidity of posttraumatic stress and functional impairment among Burundian refugee children and their parents. Eur J Psychotraumatol 10:1676005. https://doi. org/10.1080/20008198.2019.1676005

35. Song SJ, Tol W, de Jong J (2014) Indero: Intergenerational trauma and resilience between burundian former child soldiers and their children. Fam Process 53(2):239-251. https://doi.org/10.1111/ famp.12071 
36. Brislin RW, Lonner WJ, Thorndike RM (1973) Cross-cultural research methods. Wiley, New York

37. Pynoos RS, Steinberg AM (2015) The University of California, Los Angeles, Post-Traumatic Stress Disorder Reaction Index (UCLA PTSD Index) for the diagnostic and statistical manual of mental disorders, 5th edn. University of Californa, Los Angeles

38. American Psychiatric Association (2013) Diagnostic and statistical manual of mental disorders, 5th edn. Publisher, Washington, DC

39. Doric A, Stevanovic D, Stupar D et al (2019) UCLA PTSD reaction index for DSM-5 (PTSD-RI-5): a psychometric study of adolescents sampled from communities in eleven countries. Eur $\mathbf{J}$ Psychotraumatol 10(1):1605282. https://doi.org/10.1080/20008 198.2019.1605282

40. Karam EG, Fayyad JA, Farhat C et al (2019) Role of childhood adversities and environmental sensitivity in the development of post-traumatic stress disorder in war-exposed Syrian refugee children and adolescents. Br J Psychiatry 214(06):354-360. https:// doi.org/10.1192/bjp.2018.272

41. Goodman R, Meltzer H, Bailey V (2003) International review of psychiatry the Strengths and Difficulties Questionnaire: a pilot study on the validity of the self-report version the Strengths and Difficulties Questionnaire: a pilot study on the validity of the self-report version. Int Rev Psychiatry 15:173-177. https://doi. org/10.1080/0954026021000046137

42. Hoosen N, Davids EL, de Vries PJ, Shung-King M (2018) The Strengths and Difficulties Questionnaire (SDQ) in Africa: a scoping review of its application and validation. Child Adolesc Psychiatry Ment Health 12(1):1-39. https://doi.org/10.1186/s1303 4-017-0212-1

43. Panter-Brick C, Grimon MP, Eggerman M (2014) Caregiverchild mental health: a prospective study in conflict and refugee settings. J Child Psychol Psychiatry 55(4):313-327. https://doi. org/10.1111/jcpp.12167

44. Neuner F, Schauer M, Karunakara U, Klaschik C, Robert C, Elbert T (2004) Psychological trauma and evidence for enhanced vulnerability for posttraumatic stress disorder through previous trauma among West Nile refugees. BMC Psychiatry 4:34. https://doi. org/10.1186/1471-244X-4-34

45. Spirito A, Stark LJ, Williams C (1988) Development of a brief coping checklist for use with pediatric populations. J PediatrPsychol 13(4):555-574. https://doi.org/10.1093/jpepsy/13.4.555

46. Cherewick M, Doocy S, Tol W, Burnham G, Glass N (2016) Potentially traumatic events, coping strategies and associations with mental health and well-being measures among conflict-affected youth in Eastern Democratic Republic of Congo. Glob Health Res Policy 1(1):8. https://doi.org/10.1186/s4125 6-016-0007-6

47. Straus MA, Hamby SL, Finkelhor D, Moore DW, Runyan D (1998) Identification of child maltreatment with the parent-child Conflict Tactics Scales: development and psychometric data for a national sample of American parents. Child AbusNegl 22(4):249_ 270. https://doi.org/10.1016/S0145-2134(97)00174-9

48. Sierau S, White LO, Klein AM, Manly JT, von Klitzing K, Herzberg PY (2018) Assessing psychological and physical abuse from children's perspective: factor structure and psychometric properties of the picture-based, modularized child-report version of the Parent-Child Conflict Tactics Scale-Revised (CTSPCR). PLoS ONE 13(10):e0205401. https://doi.org/10.1371/journ al.pone. 0205401

49. Nkuba M, Hermenau K, Hecker T (2018) Violence and maltreatment in Tanzanian families-findings from a nationally representative sample of secondary school students and their parents. Child Abuse Negl 77:110-120. https://doi.org/10.1016/J.CHIAB U.2018.01.002
50. Gifford-Smith M (2000) People in my life. https://www.fasttrackp roject.org. Accessed 20 May 2019

51. Cook E, Greenberg M, Kusche C (1995) Attachment relationships in middle childhood. In: Presented at the biennial meeting of the Society for Research in Child Development. Indianapolis, Indiana

52. Ridenour TA, Greenberg MT, Cook ET (2006) Structure and validity of people in my life: a self-report measure of attachment in late childhood. J Youth Adolesc 35(6):1037-1053. https://doi. org/10.1007/s10964-006-9070-5

53. Moreira H, Fonseca A, Canavarro MC (2017) Assessing attachment to parents and peers in middle childhood: psychometric studies of the Portuguese version of the People in My Life Questionnaire. J Child Fam Stud 26(5):1318-1333. https://doi.org/10.1007/ s10826-017-0654-3

54. Hecker T, Fetz S, Ainamani H, Elbert T (2015) The cycle of violence: associations between exposure to violence, trauma-related symptoms and aggression-findings from Congolese refugees in Uganda. J Trauma Stress 28:448-455

55. Cohen J (1992) A power primer. Psychol Bull 112(1):155-159. https://doi.org/10.1037/0033-2909.112.1.155

56. Alisic E, Zalta AK, Van Wesel F et al (2014) Rates of posttraumatic stress disorder in trauma-exposed children and adolescents: meta-analysis. Br J Psychiatry 204(5):335-340. https://doi. org/10.1192/bjp.bp.113.131227

57 Cicchetti D, Toth SL (2016) Child maltreatment and developmental psychopathology: a multilevel perspective. In: Cicchetti D (ed) Developmental psychopathology: Vol. 3. Maladaptation and psychopathology. Wiley, Hoboken, pp 457-512

58. Lynch M, Cicchetti D (1998) An ecological-transactional analysis of children and contexts: the longitudinal interplay among child maltreatment, community violence, and children's symptomatology. Dev Psychopathol 10(2):235-257. https://doi.org/10.1017/ S095457949800159X

59. Cummings EM, Merrilees CE, Schermerhorn AC, Goeke-Morey MC, Shirlow P, Cairns E (2012) Political violence and child adjustment: longitudinal tests of sectarian antisocial behavior, family conflict, and insecurity as explanatory pathways. Child Dev 83(2):461-468. https://doi.org/10.1111/j.1467-8624.2011.01720 $\mathrm{x}$

60. Saile R, Ertl V, Neuner F, Catani C (2016) Children of the postwar years: a two-generational multilevel risk assessment of child psychopathology in northern Uganda. Dev Psychopathol 28(2):607620. https://doi.org/10.1017/S0954579415001066

61 Belsky J (2008) War, trauma and children's development: observations from a modern evolutionary perspective. Int J Behav Dev 32:260-271. https://doi.org/10.1177/0165025408090969

62. Dubow EF, Huesmann LR, Boxer P (2009) A social-cognitiveecological framework for understanding the impact of exposure to persistent ethnic-political violence on children's psychosocial adjustment. Clin Child FamPsychol Rev 12(2):113-126. https:// doi.org/10.1007/s10567-009-0050-7

63. Qouta S, Punamäki R-L, Miller T, El-Sarraj E (2008) Does war beget child aggression? Military violence, gender, age and aggressive behavior in two Palestinian samples. Aggress Behav 34(3):231-244. https://doi.org/10.1002/ab.20236

64. Keresteš G (2006) Children's aggressive and prosocial behavior in relation to war exposure: testing the role of perceived parenting and child's gender. Int J Behav Dev 30(3):227-239. https://doi. org/10.1177/0165025406066756

65. McMahon SD, Todd NR, Martinez A et al (2013) Aggressive and prosocial behavior: community violence, cognitive, and behavioral predictors among urban African American youth. Am J Community Psychol 51(3-4):407-421. https://doi.org/10.1007/s1046 4-012-9560-4

66. Staub E, Vollhardt J (2008) Altruism born of suffering: the roots of caring and helping after victimization and other trauma. Am 
J Orthopsychiatry 78(3):267-280. https://doi.org/10.1037/a0014 223

67. Gneezy A, Fessler DMT (2012) Conflict, sticks and carrots: war increases prosocial punishments and rewards. Proc $\mathrm{R}$ Soc B BiolSci 279(1727):219-223. https://doi.org/10.1098/ rspb.2011.0805

68. Silke C, Brady B, Boylan C, Dolan P (2018) Factors influencing the development of empathy and pro-social behaviour among adolescents: a systematic review. Child Youth Serv Rev 94:421-436. https://doi.org/10.1016/j.childyouth.2018.07.027

69. Mueller-Bamouh V, Ruf-Leuschner M, Dohrmann K, Schauer M, Elbert T (2016) Are experiences of family and of organized violence predictors of aggression and violent behavior? A study with unaccompanied refugee minors. Eur J Psychotraumatol 7(1):27856. https://doi.org/10.3402/ejpt.v7.27856

70. Huijts I, Kleijn WC, van Emmerik AAP, Noordhof A, Smith AJM (2012) Dealing with man-made trauma: the relationship between coping style, posttraumatic stress, and quality of life in resettled, traumatized refugees in the Netherlands. J Trauma Stress 25(1):71-78. https://doi.org/10.1002/jts.21665

71. Shisana O, Celentano DD (1987) Relationship of chronic stress, social support, and coping style to health among Namibian refugees. SocSci Med 24(2):145-157. https://doi.org/10.1016/02779536(87)90247-4

72. Seglem KB, Oppedal B, Roysamb E (2014) Daily hassles and coping dispositions as predictors of psychological adjustment: a comparative study of young unaccompanied refugees and youth in the resettlement country. Int J Behav Dev. https://doi. org/10.1177/0165025414520807

73 Woltin KA, Sassenberg K, Albayrak N (2018) Regulatory focus, coping strategies and symptoms of anxiety and depression: a comparison between Syrian refugees in Turkey and Germany. PLoS ONE. https://doi.org/10.1371/journal.pone.0206522

74. Criss MM, Pettit GS, Bates JE, Dodge KA, Lapp AL (2002) Family adversity, positive peer relationships, and children's externalizing behavior: a longitudinal perspective on risk and resilience. Child Dev 73(4):1220

75. Lansford JE, Criss MM, Laird RD et al (2011) Reciprocal relations between parents' physical discipline and children's externalizing behavior during middle childhood and adolescence. Dev Psychopathol 23(1):225-238. https://doi.org/10.1017/S0954 579410000751

76 Layous K, Nelson SK, Oberle E, Schonert-Reichl KA, Lyubomirsky S (2012) Kindness counts: prompting prosocial behavior in preadolescents boosts peer acceptance and well-being. Krueger F, ed. PLoS ONE 7(12):e51380. https://doi.org/10.1371/journ al.pone. 0051380

77. Gadeberg AK, Norredam M (2016) Urgent need for validated trauma and mental health screening tools for refugee children and youth. Eur Child Adolesc Psychiatry 25(8):929-931. https://doi. org/10.1007/s00787-016-0837-2

78. Cohen JA, Mannarino AP (2008) Trauma-focused cognitive behavioural therapy for children and parents. Child AdolescMent Health 13(4):158-162. https://doi.org/10.111 1/j.1475-3588.2008.00502.x

79 Yule W, Dyregrov A, Raundalen M, Smith P (2013) Children and war: the work of the Children and War Foundation. Eur J Psychotraumatol. https://doi.org/10.3402/ejpt.v4i0.18424

80. O'Callaghan P, McMullen J, Shannon C, Rafferty H, Black A (2013) A randomized controlled trial of trauma-focused cognitive behavioral therapy for sexually exploited, war-affected congolese girls. J Am Acad Child Adolesc Psychiatry 52(4):359-369. https ://doi.org/10.1016/j.jaac.2013.01.013

81. Sarkadi A, Ådahl K, Stenvall E et al (2018) Teaching recovery techniques: evaluation of a group intervention for unaccompanied refugee minors with symptoms of PTSD in Sweden. Eur Child Adolesc Psychiatry 27(4):467-479. https://doi.org/10.1007/s0078 7-017-1093-9 